\title{
Fabrication of Degradable Intervertebral Fusion with Good Mechanical Properties and Biocompatibility Based on 3D Printing Technology
}

\author{
WANG Qiang ${ }^{1,2, a}$, ZHENG Xiongfei ${ }^{2, b}$ and WANG Heran ${ }^{2}$ \\ ${ }^{1}$ School of mechanical engineering, ShenYang Ligong University, Shenyang, China \\ ${ }^{2}$ State Key Laboratory of robotics, Shenyang Institute of automation, Chinese Academy of Sciences, \\ Shenyang, China \\ a15840211950@163.com, ${ }^{\mathrm{b}}$ Zhengxiongfei@sia.com
}

\begin{abstract}
Keywords: 3D printing technology; Intervertebral fusion stent; Mechanical properties; technological method.

Abstract. In this paper, the manufacture method of degradable intervertebral fusion stent with good mechanical properties and biocompatibility based on 3D printing technology is researched. Firstly, the mixing process of synthetic macromolecule and bioceramic powder is studied. And develop a high temperature stirring grinding device and realize arbitrary proportion of composite materials. Secondly, the intervertebral fusion model is processed and the print path is optimized based on discrete allocation algorithm of modeling section of cylindrical structure and print trajectory optimization algorithm. Then, the influence of printing temperature and printing layer on the bonding strength of the stent is studied, which provides a theoretical basis for the reasonable parameters setting. In addition, the composite for stent is studied, with different materials in different ratios are mixed and compressive strength and compressive modulus of each stent are tested. The ratio of composite with good mechanical strength is obtained. Finally, the intervertebral cage stent is constructed with good mechanical properties and biocompatibility.
\end{abstract}

\section{Introduction}

Intervertebral fusion is a common technique for the treatment of instability and slippage of the spine. The mechanical stability of the spine is reconstructed by creating bony fusion between the upper and lower segments. More than 200 thousand patients undergo intervertebral fusion only America each year [1]. In the early stage, spinal fusion mainly used self-skeletal, but it is prone to problems about graft and limited amounts of bone. At present, the intervertebral fusion is mainly made of titanium alloy, which reduces the problem of autologous transplantation, but there are some problems such as too high modulus of elasticity and poor biocompatibility [2]. The pore structure is not good enough by traditional machining methods at the same time. Therefore, it is necessary to develop a biodegradable intervertebral fusion with good mechanical properties and biocompatibility. At present, there has been some research on the degradable intervertebral fusion. T. Li, in The Fourth Military Medical University, carried out some studies on the TCP/PCL composite stent, and made the fusion with lower TCP content and carried out animal experiments. The fabrication of composite is studied in this paper $[3,4]$. The composite materials PCL, TCP and PLGA in different proportions are fabricated, and the mechanical properties of the different material stent are tested, which verifies the effect of TCP and PLGA on the mechanical strength [5, 6]. At the same time, the printing parameters that affect the bond strength of the stent are studied, which provides theoretical support for the parameter setting of stent printing. The study on degradable intervertebral fusion materials research and development has guiding significance, providing effective methods for the fabrication with good mechanical properties of the fusion and laying the foundation for the future.

\section{Materials and Methods.}

Fabrication of Composite Materials. In this experiment, synthetic macromolecule materials PCL, PLGA and TCP (refer with: Table 1) are mixed at different ratios to fabricate composite materials of PCL:TCP=3:1, PCL:TCP=5:5 and PCL:TCP:PLGA=1:2:1. The mix of synthetic macromolecule and 
bio-ceramics is achieved by high temperature stirring and grinding devices. In the process of material mixing, PCL, PLGA and other polymer materials are firstly placed in a high temperature grinding mixer to be melted and stirred(refer with: Fig.1 (a)). At the same time, a suitable amount of bio-ceramic material TCP is sprayed uniformly into the high-temperature mixer by an atomizer to be polished and blended evenly with the synthetic macromolecule. Then, the material is extruded through the outlet below the agitator to form a bar and wire material that can be directly used for printing (refer with: Fig.1 (b)).

(a)

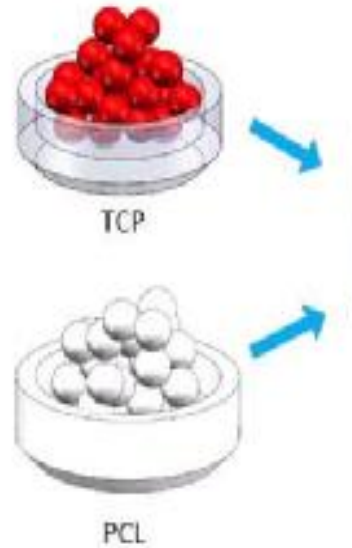

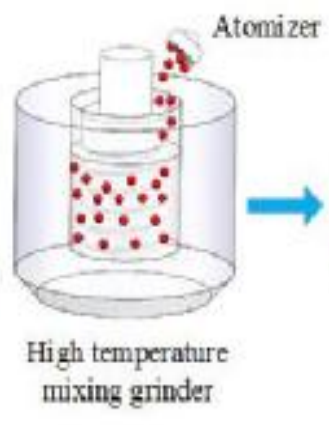

mixing grinder

(a) Schematic Diagram of The Preparation Process of Composite Materials;

Fig. 1 -Schematic Diagram of Composite Material Preparation Process and Composite Bone Material

Table 1 -Basic Properties of Materials

$\begin{array}{ccccc}\text { Material } & \text { Full name } & \begin{array}{c}\text { Glass transition } \\ \text { temperature }\left({ }^{\circ} \mathrm{C}\right)\end{array} & \begin{array}{c}\text { Melting } \\ \text { point }\left({ }^{\circ} \mathrm{C}\right)\end{array} & \text { Source of purchase } \\ \text { PCL } & \text { Poly }\left({ }^{\varepsilon} \text {-caprolactone }\right) & -60 & >63 & \begin{array}{c}\text { Shenzhen Polymtek Biomaterials } \\ \text { Co., Ltd }\end{array} \\ \text { TCP } & \beta \text {-TCP } & - & - & \begin{array}{c}\text { Shanghai Bio-Lu Biomaterials } \\ \text { Co., Ltd }\end{array} \\ \text { PLGA } & \text { Poly(L-lactide) } & 60 \sim 65 & 175 \sim 185 & \begin{array}{c}\text { Shenzhen Polymtek Biomaterials } \\ \text { Co., Ltd }\end{array}\end{array}$

3D Printing System. 3D printing system mainly includes high-precision three freedom motion platform, high-precision extrusion system, temperature control system and CAD/CAM system (refer with: Fig.2). The three degree of freedom motion platform is controlled by the motion controller and can move in the XYZ direction. The 3D model can be constructed. High precision extrusion system is mainly composed of controller, encoder and high precision nozzle. It adopts screw extrusion method. The temperature control system mainly controls the temperature of the nozzle accurately. CAD/CAM system mainly completes 3D modeling, slicing and printing trajectory optimization. The model is sliced and layered based on discrete allocation algorithm of modeling section of cylindrical structure. At the same time, the 3D print path planning algorithm is used to optimize the print trajectory. Finally, the $\mathrm{G}$ file used for printing is generated with reference to the print parameters. 


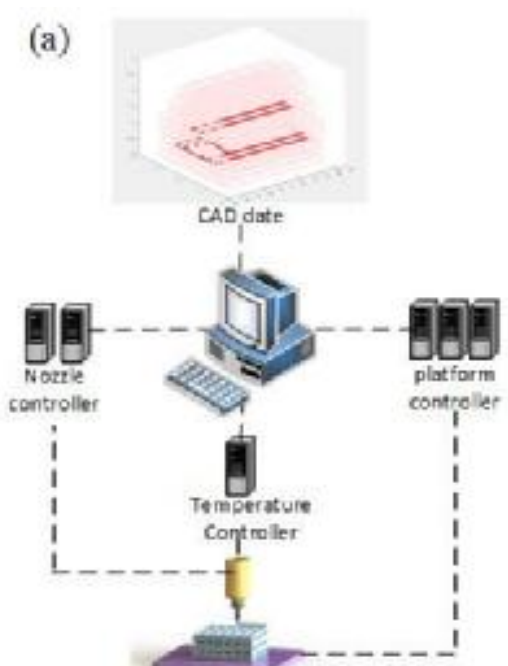

(b)

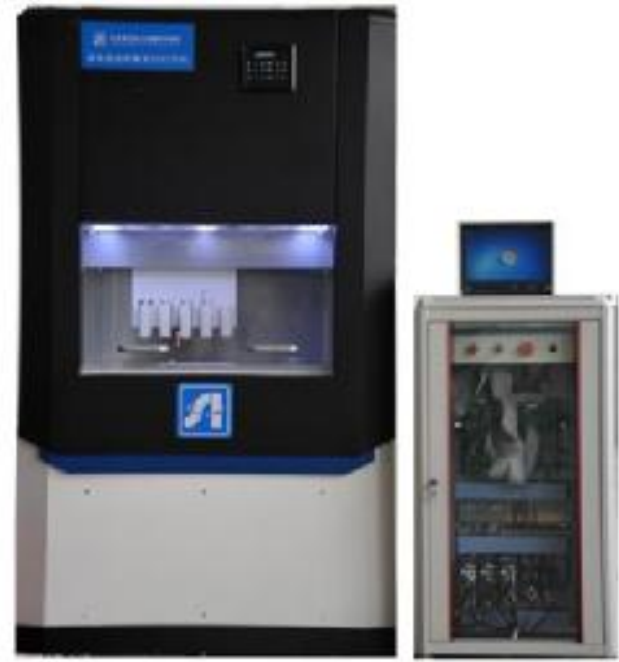

(c)
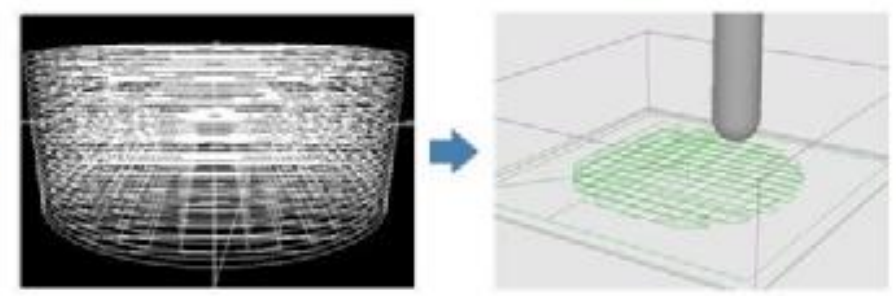

Fig.2 -3D Printing System and Model Processing. (a) 3D Print System Architecture Diagram; (b) 3D Printer Physical Diagram; (c) Model Processing.

Technological Experiment. In the experiment of printing PCL, the temperature of the nozzle is independent variable, and other parameters are constant. The temperature of the nozzle is 90,105 and 135, respectively (refer with: Table 2). The six same stents are printed at each temperature. The bracket is the cube of the $4 \times 4 \times 6 \mathrm{~mm}$. The bonding force of PCL bracket at different temperatures is tested by mechanical testing device (refer with: Fig.3) and recorded the test data.

At the same time, the influence of the layer thickness on the cohesive force is explored, In the experiment of printing PCL, the temperature of the nozzle is independent variable, and other parameters are constant. The layer thickness is $320 \mathrm{um}, 260 \mathrm{um}$ and $200 \mathrm{um}$, respectively (refer with: Table 3). The six same stents are printed at each temperature. The bracket is the cube of the $4 \times 4 \times 6 \mathrm{~mm}$. The bonding force of PCL bracket at different temperatures is tested by mechanical testing device (refer with: Fig.3) and recorded the test data.

During the experiment of printing different materials, the rods of different materials are respectively loaded into the nozzle, and reasonable print parameters are set according to the material characteristics, and an intervertebral fusion bracket is constructed by the 3D printing system. The compressive strength and elastic modulus of the cage are analyzed by mechanical test, and the forming structure of the fusion is analyzed.

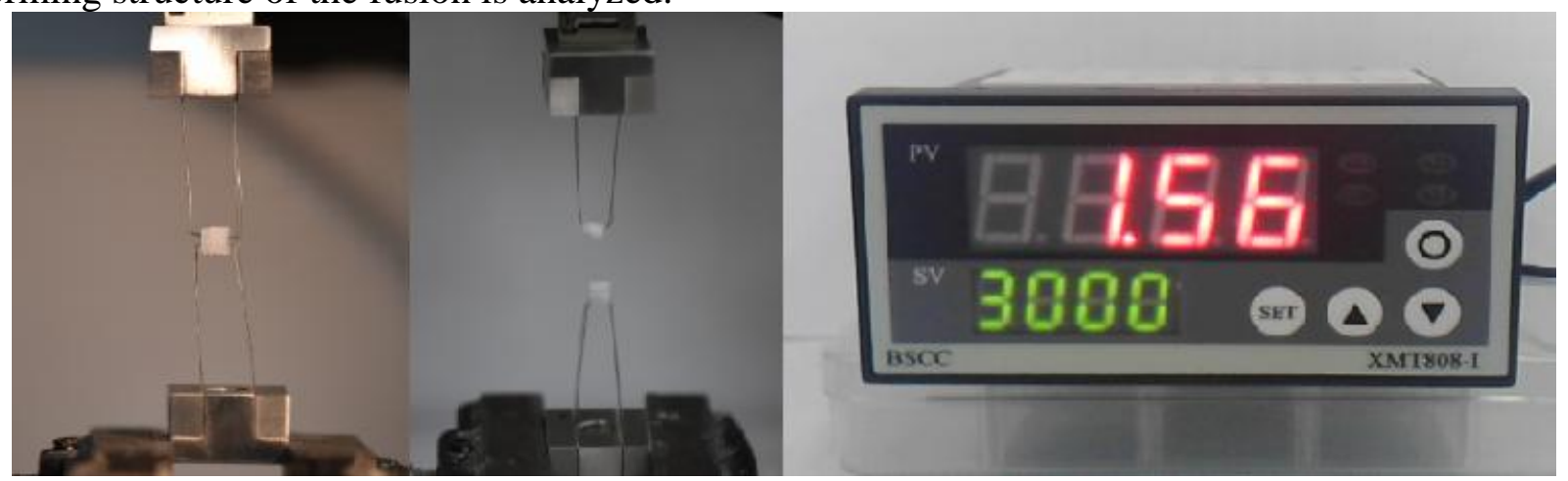

Fig.3 -Mechanical Testing Process 
Table2. Print parameter settings

\begin{tabular}{cccccc}
\hline Material & $\begin{array}{c}\text { Layer thickness } \\
(\text { um })\end{array}$ & $\begin{array}{c}\text { Nozzle } \\
\text { temperature } \\
\left({ }^{\circ} \mathrm{C}\right)\end{array}$ & $\begin{array}{c}\text { Line } \\
\text { width } \\
(\mathrm{mm})\end{array}$ & $\begin{array}{c}\text { Extrusion } \\
\text { speed } \\
(\mathrm{mm} \mathrm{s}-1)\end{array}$ & $\begin{array}{c}\text { Scan } \\
\text { velocity } \\
(\mathrm{mm} \mathrm{s}-1)\end{array}$ \\
\hline \multirow{3}{*}{ PCL } & 260 & 90 & 1.0 & 0.018 & 4.0 \\
& 260 & 105 & 1.0 & 0.018 & 4.0 \\
& 260 & 135 & 1.0 & 0.018 & 4.0
\end{tabular}

Table3. Print parameter settings

\begin{tabular}{cccccc}
\hline Material & $\begin{array}{c}\text { Layer thickness } \\
(\mathrm{um})\end{array}$ & $\begin{array}{c}\text { Nozzle } \\
\text { temperature } \\
\left({ }^{\circ} \mathrm{C}\right)\end{array}$ & $\begin{array}{c}\text { Line } \\
\text { width } \\
(\mathrm{mm})\end{array}$ & $\begin{array}{c}\text { Extrusion } \\
\text { speed } \\
(\mathrm{mm} \mathrm{s}-1)\end{array}$ & $\begin{array}{c}\text { Scan } \\
\text { velocity } \\
(\mathrm{mm} \mathrm{s}-1)\end{array}$ \\
\hline \multirow{3}{*}{ PCL } & 320 & 90 & 1.0 & 0.018 & 4.0 \\
& 260 & 90 & 1.0 & 0.018 & 4.0 \\
& 200 & 90 & 1.0 & 0.018 & 4.0 \\
\hline
\end{tabular}

Table4. Print parameter settings

\begin{tabular}{cccccc}
\hline Material & $\begin{array}{c}\text { Layer } \\
\text { thickness } \\
(\mathrm{um})\end{array}$ & $\begin{array}{c}\text { Nozzle } \\
\text { temperature } \\
\left({ }^{\circ} \mathrm{C}\right)\end{array}$ & $\begin{array}{c}\text { Line } \\
\text { width } \\
(\mathrm{mm})\end{array}$ & $\begin{array}{c}\text { Extrusion } \\
\text { speed } \\
(\mathrm{mm} \mathrm{s}-1)\end{array}$ & $\begin{array}{c}\text { Scan velocity } \\
(\mathrm{mm} \mathrm{s}-1)\end{array}$ \\
\hline PCL & 320 & 90 & 1.0 & 0.018 & 4.0 \\
PCL:TCP=3:1 & 320 & 125 & 1.0 & 0.020 & 4.0 \\
PCL:TCP=5:5 & 320 & 140 & 1.0 & 0.024 & 4.0 \\
PCL:TCP:PLGA & 320 & 192 & 1.0 & 0.020 & 4.0 \\
$=1: 2: 1$ & & & & & \\
\hline
\end{tabular}

\section{Results.}

Influence of Printing Parameters on Bond Strength of Stent. The change curve of the bond strength with the print temperature is shown in the figure (refer with: Fig.4 (a)). The experimental results show that the bonding strength increases with the increase of the printing temperature in a certain range of temperature. The curve of the bond strength of the support with the print height is shown in the figure (refer with: Fig.4 (b)). In a certain height setting range, with the increasing of print height, cohesive force support decreases, but with reduced print height, between the stent layer and layer pore decreases, the overall poor stent structure. Therefore, the reasonable printing temperature and the setting of the printing height can not only ensure the good bonding strength, but also ensure the good forming structure of the stent.

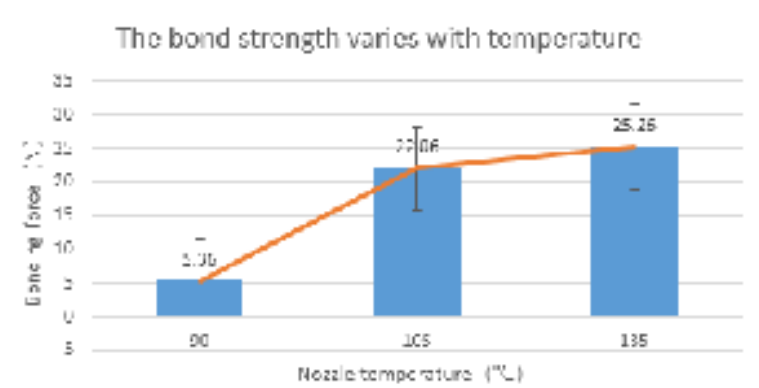

(a) -Curves of Bond Strength Versus Temperature

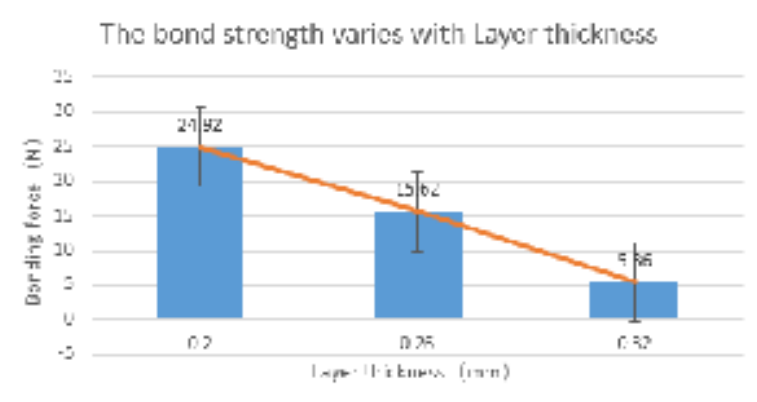

(b) -Curve of the Bond Strength of the Support with the Player thickness

Fig. 4 - Mechanical Test Curve of Stent

Forming Result of Intervertebral Fusion. The printing process of the intervertebral fusion is shown (refer with: Fig.5 (a)). The intervertebral fusion of (b), (c), (d) and (e) are PCL, PCL:TCP=3:1, 
PCL:TCP=5:5, and PCL:TCP:PLGA=1:2:1 respectively. The results show that the reasonable material preparation process and printing parameters make the intervertebral fusion have better molding effect and high porosity. Fig.(f) and (g) are the compressive strength and modulus of the fusion for different materials, respectively. The results show that the single PCL material with the lower compressive strength and modulus of elasticity. With the increasing proportion of TCP, the compressive strength and elastic modulus are increasing. When the material ratio is PCL:TCP:PLGA=1:2:1, the stent has the highest compressive strength and elastic modulus.

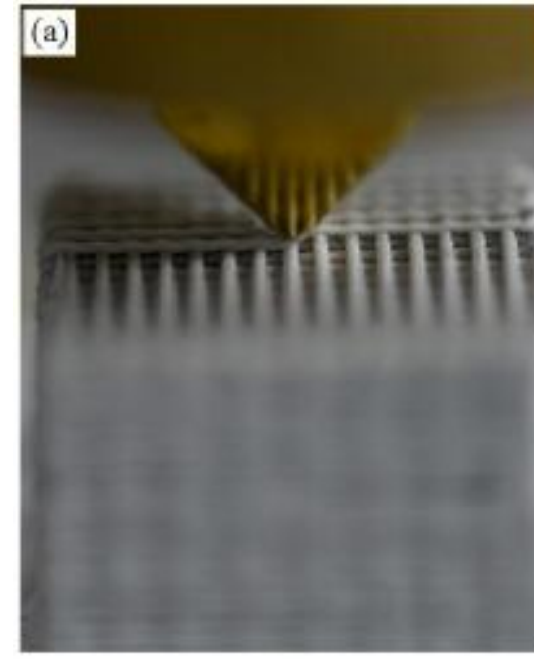

(f) Compressive strength varies with material

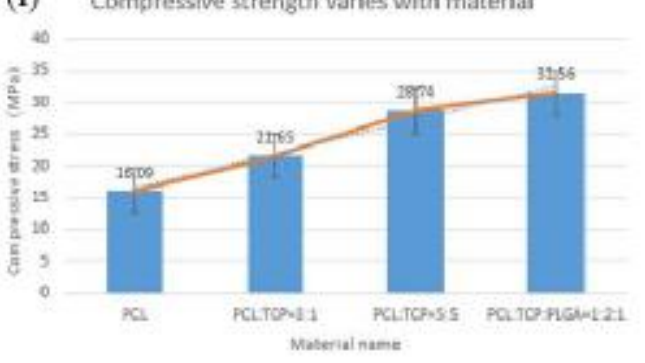

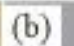

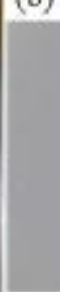

\section{(d)}

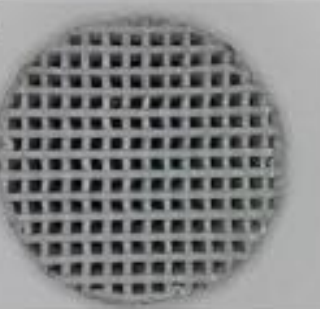

(c)

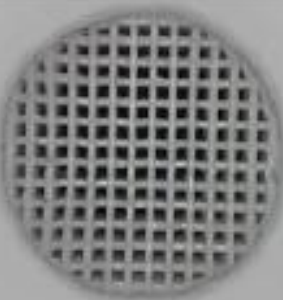

(e)

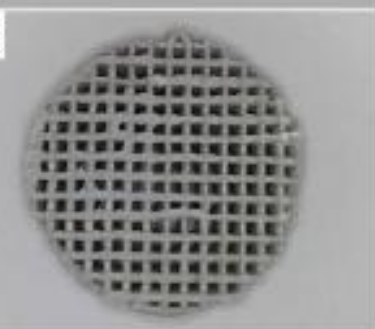

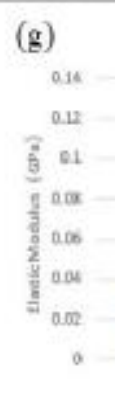

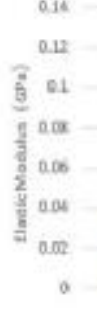

Elastic Modulus varies with material

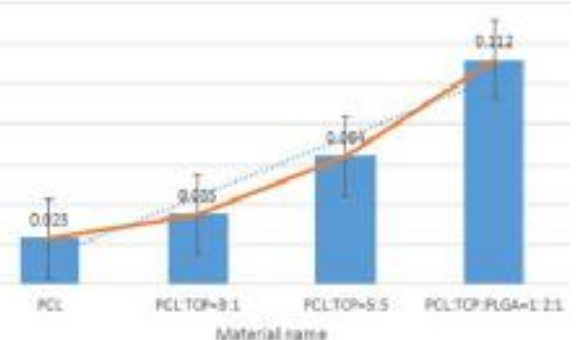

(a) The Procedure of Stent ; (b) PCL Stent; (c) PCL:TCP=3:1 Stent; (d) PCL:TCP=5:5 Stent; (e) PCL:TCP:PLGA=1:2:1 Stent.(f) The Compressive Strength Varies with the Material; (g) The Modulus Varies with the Material

Fig. 5 -The Printing Process, Forming Structure and Mechanical Test Results of Intervertebral Fusion

Animal Experiment of Intervertebral Fusion. The CT image of the intervertebral fusion implanted in goats is shown (refer with: Fig.6). The material for the intervertebral fusion is PCL:TCP:PLGA=1:2:1. The experimental results show that the intervertebral fusion has good biocompatibility and less deformation under compression. It is proved that the stent has relatively good mechanical properties.

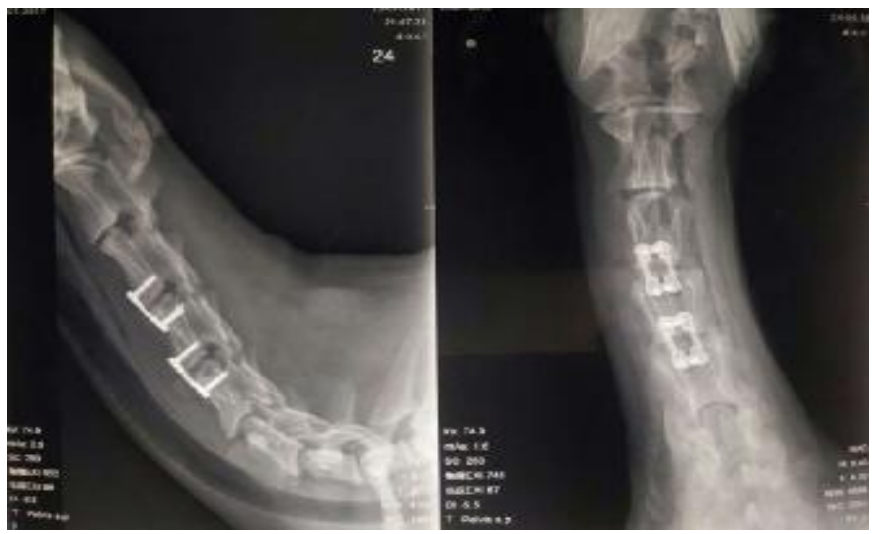

Fig.6-Animal Experiment of Intervertebral Fusion 


\section{Summary}

Based on the research of bio 3D printing technology, through the research of material ratio, composite material fabrication and printing parameter setting method, a degradable stent for intervertebral fusion was successfully constructed with the help of 3D bio printing system, discrete allocation algorithm of modeling section of cylindrical structure and print trajectory optimization algorithm. The stent has good biocompatibility, but also has similar mechanical properties and mechanism of pore and natural bone. At the same time, this paper explores a set of effective technological method of fabrication of composite material and bone stent print process. The study laid the foundation and it has important clinical significance.

\section{References}

[1] Li Y. Design, preparation and in vivo study of PCL-TCP cervical intervertebral disc cage [D]. The Fourth Military Medical University. 2014.

[2] Lee J S, Hong J M, Jung J W, et al. 3D printing of composite tissue with complex shape applied to ear regeneration.[J]. Biofabrication, 2014, 6(2):024103-024103.

[3] Lee J S, Hong J M, Jung J W, et al. 3D printing of composite tissue with complex shape applied to ear regeneration[J]. Biofabrication, 2014, 6(2):024103.

[4] Arai K, Iwanaga S, Toda $\mathrm{H}$, et al. Three-dimensional inkjet biofabrication based on designed images.[J]. Biofabrication, 2011, 3(3):034113-034113.

[5] Shim J H, Kim J Y, Park M, et al. Development of a hybrid scaffold with synthetic biomaterials and hydrogel using solid freeform fabrication technology.[J]. Biofabrication, 2011, 3(3):págs. 291-312.

[6] Duan B, Hockaday L A, Kang K H, et al. 3D bioprinting of heterogeneous aortic valve conduits with alginate/gelatin hydrogels.[J]. Journal of Biomedical Materials Research Part A, 2013. 